\title{
Psicología y enseñanza de la Lengua
}

\author{
IGNASI VILA
}

\author{
Universidad de Barcelona
}

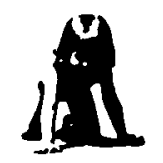

\section{Resumen}

El artículo versa sobre las relaciones entre psicología y didáctica en relación con la enseñanza de la lengua. Así, se defiende que la psicolingǘstica no puede prescribir los procesos de enseñanza-aprendizaje de la lengua, aunque aporta elementos de reflexión, especialmente los referidos a las competencias implicadas en el uso del lenguaje. Se discute también el papel de la didáctica para concluir que aquello que se debe investigar son los mecanismos de influencia educativa que promueven el aprendizaje de los procedimientos implicados en el uso del lenguaje. Desde este punto de vista, se propone una convergencia entre la psicología educativa y la didáctica.

Palabras clave: Psicolingüística evolutiva; Didáctica de la lengua; Enseñanza de la lengua; Psicología de la educación; Ajuste de la ayuda pedagógica.

\section{Psychology and Language Instruction}

\section{Abstract}

This article deals with relations between psychology and didactics with respect to language teaching. It mantains that psycholinguistics can not prescribe language teaching-learning processes, though it contributes elements of thought, especially those referred to the skills involved in the use language. The role of didactics is also discussed in order to conclude that what must be investigated are the mechanisms of educational influence promoted by learning the procedures involved in the use of language. From this point of view, a convergence between Educational Psychology and Didactics is proposed.

Key words: Developmental Psychology; Language Didactics; Language teaching; Educational Psychology; Adjustment of teacher's help.

Correspondencia con autor: Universitat de Barcelona. Institut de Ciències de l'Educació. Angels, 18. 08001 Barcelona. 


\section{INTRODUCCION}

Las relaciones entre didáctica y psicología han sido objeto de amplios debates en los últimos años. Soy de los que piensa que la psicología de la educación puede ayudar a conceptualizar, planificar y mejorar los procesos de enseñanzaaprendizaje, pero también pienso que si no se tiene en cuenta el contenido concreto a enseñar y aprender no es posible comprender los procesos de enseñanzaaprendizaje en su totalidad. Por eso, en el empeño de mejorar dichos procesos deben trabajar mano a mano tanto los psicólogos como las personas que trabajan e investigan en el ámbito de las didácticas específicas. Es decir, no creo en una psicología prescriptiva que, desde su conocimiento, pueda especificar y determinar la forma en que deben transcurrir los procesos de enseñanza-aprendizaje. De hecho, al menos en el campo de la lengua, cuando desde la psicología se ha prescrito (método audiolingual, método directo, etc.) cómo enseñar el lenguaje, el fracaso ha sido total. Por eso, como psicólogo que soy y, por tanto, desconocedor en gran medida de muchas de las aportaciones de la didáctica de la lengua, en lo que sigue sólo pretendo realizar algunas consideraciones a tener en cuenta desde la psicolingǘstica en la enseñanza de la lengua.

\section{PSICOLOGIA Y ENSEÑANZA DE LA LENGUA}

No es este el lugar para hacer una revisión completa de las relaciones que han existido entre psicología y enseñanza de la lengua. Sin embargo, sí que creo que es importante resaltar algunos rasgos que, a mi entender, continúan existiendo en la actualidad.

En primer lugar, mi experiencia en el trabajo conjunto con profesores y profesoras de la Enseñanza Primaria y Secundaria me lleva a pensar en el mantenimiento de concepciones prescriptivas sobre la psicología o, en este caso, la psicolingüística. Ciertamente, durante muchos años se ha hecho creer a los enseñantes en la existencia de conocimientos psicológicos omnipotentes que podían dar cuenta sin problemas, bien de los mecanismos implicados en el aprendizaje, bien de los mecanismos implicados en la construcción del conocimiento. Es decir, se presentaba una disociación entre aquello que el alumno debía conocer y cómo llegaba a conocerlo. La psicología no era pertinente en el ámbito del establecimiento de los contenidos, al igual que la ciencia específica, en este caso la lingüística, no tenía nada que decir sobre cómo se adquirían los conocimientos lingüísticos. Justamente, en este planteamiento la didáctica aparecía como el puente que, sin cuestionar los conocimientos provenientes bien de la psicología, bien de la lingǘstica, proporcionaba la metodología (métodos, secuenciaciones, ejercicios, etc.) para actuar en el ámbito del aula. Desgraciadamente, creo que esta concepción continúa presente y, por tanto, no es extraño escuchar preguntas sobre la edad en que un escolar puede adquirir tal o tal otra cosa con el objeto de determinar si se le puede enseñar o no. Ello se complica aún más con mi segunda apreciación. Creo, también, que entre los enseñantes domina una visión muy particular de la ciencia: a ésta se la ve también como omnipotente, como poseedora de la verdad y, por tanto, la relación que una parte importante del profesorado establece con la lingüística es una relación acrítica que sólo persigue, en el mejor de los casos, la búsqueda del mejor modelo, el que provee mayor certeza sobre los hechos lingüísticos, modelo que, evidentemente, es el que presentan a los escolares para 
su aprendizaje. No es extraño, por ejemplo, comprobar actualmente la existencia de actitudes que intentan sustituir las descripciones estructurales del aparato formal del lenguaje por descripciones provenientes de las gramáticas del discurso sin que exista una reflexión sobre el rol que debe jugar en la enseñanza de la lengua el dominio de los mecanismos formales implicados en el lenguaje. Pero, a la vez, junto a una enseñanza de la lengua formalista, descontextualizada e inútil, la mayor parte de las veces, existe un consenso sobre los objetivos lingüísticos a conseguir a lo largo de la enseñanza obligatoria. Así, cuando se interroga a los enseñantes acostumbran a decir que el objetivo de la enseñanza de la lengua remite al dominio de las cuatro destrezas implicadas en el uso del lenguaje (escuchar, hablar, leer y escribir). Ello sitúa al área de lengua fundamentalmente como un área instrumental. Ciertamente, dicho consenso no es ajeno a la propia práctica de la enseñanza de la lengua. Es decir, en los últimos veinte años, un sector de profesionales de la enseñanza de la lengua han reivindicado los aspectos comunicativos implicados en el uso del lenguaje frente a los análisis linguiísticos tradicionales. Sin embargo, los resultados de las prácticas derivadas de estas concepciones no son satisfactorios. De hecho, como señala Camps (1990), en esta tradición existe una notable confusión entre los objetivos a conseguir y las prácticas a realizar, convirtiéndose la enseñanza de la lengua en un conjunto de actividades que se siguen la una a la otra de forma espontánea sin que exista clara conciencia de lo que deben aprender los alumnos y en relación a qué tipo de objetivos. Talleres literarios, textos libres, asambleas, debates, lectura expresiva, etc., se suceden a lo largo de la enseñanza obligatoria como actividades de lengua sin que, en ningún momento, ni profesor ni alumno sepan lo que se pretende con ellas más allá de su simple realización.

De hecho, se continúa creyendo que el lenguaje «crece» de la misma forma que a los pájaros les crecen las alas. Sólo hace falta colocar a las personas en situaciones comunicativas para que las capacidades linguísticas, supuestamente preexistentes, se desplieguen y se realicen según su diversidad de usos. Por eso, concepciones como las de Krashen y Terrell en el ámbito de la enseñanza de segundas lenguas, o frases hechas como que a hablar se aprende hablando y a escribir, escribiendo, han tenido tanto éxito en los últimos años y se han convertido en el leivmotiv de numerosas programaciones y actividades. No cabe decir que las ideas de Chomsky y un largo etcétera de trabajos de psicolingüística han abonado esta concepción. Curiosamente, personas que se llenan la boca con términos relativos al enfoque comunicativo y rechazan la existencia de aspectos innatos en el proceso de adquisición del lenguaje, cuando traducen sus ideas a la enseñanza de la lengua caen prisioneras de esta concepción y remiten las actividades de enseñanza-aprendizaje de la lengua a un supuesto proceso «natural» en el que se presupone la existencia de una continuidad funcional a lo largo de todo el desarrollo.

No es este tampoco el lugar para discutir los mecanismos implicados en el proceso de adquisición del lenguaje, pero valga la siguiente cita para situar con claridad las implicaciones de lo innato para la enseñanza de la lengua.

«tomemos dos individuos que tengan el mismo conocimiento del español... estos dos individuos pueden diferir - y característicamente diferirán mucho- en su capacidad de usar la lengua. El uno puede ser un gran poeta, y el segundo puede usar una lengua perfectamente pedestre y expresarse con clichés. Por sus características, dos individuos que comparten el mismo conocimiento de un mismo idioma se incli- 
narán a decir cosas muy diferentes en ocasiones dadas. De aquí que sea difícil comprender cómo se puede identificar el conocimiento con la destreza y aún menos con la disposición al comportamiento» (Chomsky, 1988: 18).

Por tanto, aun admitiendo aspectos innatos relativos a la competencia lingiústica, ello poco tiene que ver con lo que estamos discutiendo. En otras palabras, aceptar la existencia de competencias lingüísticas semejantes entre todos los hablantes de una determinada comunidad lingüística no es óbice para reconocer, a la vez, la existencia de enormes diferencias en el ámbito de la realización, diferencias que no son innatas, sino que remiten al dominio de los procedimientos implicados en el uso del lenguaje, el cual no «crece», sino que se aprende si alguien que ya lo posee lo enseña. Por eso, las cuestiones relevantes a discutir en el ámbito de la enseñanza de la lengua no se refieren a los mecanismos generales implicados en el proceso de adquisición del lenguaje, sino a los mecanismos de influencia educativa implicados en el aprendizaje de los procedimientos relativos al uso del lenguaje. Por descontado, ello no significa, como veremos más adelante, que determinados conocimientos psicolingüísticos relativos al uso del lenguaje no sean relevantes para la planificación y mejora de los procesos de enseñanza-aprendizaje, pero, en cualquier caso, dichos conocimientos deben estar al servicio de algo más general que remite a la forma de planificar de manera intencional qué, cómo y cuándo debe aprender sobre la lengua un escolar para mejorar sus usos orales y escritos.

\section{ALGUNAS CUESTIONES A TENER EN CUENTA EN LA ENSEÑANZA DE LA LENGUA}

En primer lugar, ciertamente el lenguaje se emplea en situaciones comunicativas inter- $o$ intrapersonales. Ello implica que el lenguaje se emplea cuando alguien tiene algo que decir a otro o a sí mismo y, a la vez, existe comunicación cuando el interlocutor se muestra interesado en aquello que le dicen. Esta consideración, que puede sonar a perogrullo, creo que tiene importantes implicaciones en la enseñanza de la lengua.

Así, no existe lenguaje al margen de las intenciones, al margen de aquello que se quiere decir o, en otras palabras, el lenguaje está en estrecha conexión con el pensamiento y, por tanto, reducir la enseñanza de la lengua a sus aspectos expresivos sin reconocer dicha relación conduce directamente al espontaneísmo. De lo que se trata, por el contrario, es de enseñar a decir mejor aquello que ya se dice o a entender mejor aquello que ya se entiende. Es habitual escuchar que si no se sabe decir una cosa es porque en el fondo no se sabe; es decir, se establece una relación directa entre lo que se sabe y cómo se dice; sin embargo, dicha relación es más complicada o, en otras palabras, saber una cosa no significa directamente saber cómo decirla o reconocerla. Desgraciadamente, en la escuela muchas veces se habla por hablar o se lee por leer, de modo que ni el alumno tiene nada que decir realmente, ni el profesor está interesado en saber si tiene algo que decir y, por tanto, la realización de la actividad se limita al reconocimiento de aspectos formales (mucho o poco léxico, uso de sinónimos, producciones sintácticamente correctas, velocidad lectora, etc.) por parte de uno y de otro. En el ámbito de la escritura, valga el ejemplo por la época en la que estamos, una parte importante de los alumnos y las alumnas de Catalunya repite 
la leyenda de Sant Jordi en sus más variadas formas (dibujo, cuento, poesía, redacción, etc.) o escribe sobre la primavera, año tras año, a lo largo de toda la EGB sin que, de hecho, nadie se pregunte si les interesa o si tienen algo que decir (por otra parte, difícil de creer, después de cuatro, cinco o seis años de hacer exactamente lo mismo). Igualmente, bajo el paraguas de la creatividad, se prima un tipo de actividades de lengua escrita y se olvida, por ejemplo, la enseñanza de la lengua escrita que emplea el propio contexto escolar.

En fin, creo que la enseñanza de la lengua debe realizarse en relación a actividades en las que el alumno tenga algo que decir a alguien, de otra forma el contexto comunicativo que se crea es falso y la actividad que realiza el alumno se convierte en sí misma en el objetivo de la enseñanza de la lengua, de modo que el escuchar, el hablar, el leer y el escribir devienen en actividades sin sentido para aquel que las realiza.

Una segunda cuestión remite al tipo de usos lingüísticos que puede y debe enseñar la escuela. Si pensamos en el lenguaje desde una perspectiva instrumental, lo primero que acude a nuestra mente es que la vida social de las personas está regulada a través del lenguaje. Es decir, la educación formal no es el único lugar donde se desarrolla conocimiento lingüístico. Por el contrario, los niños y las niñas, ya desde la cuna, están inmersos en contextos informales en los que deben negociar procedimientos lingüísticos y no lingüísticos para asegurar sus intercambios comunicativos en el ámbito de la regulación de sus conductas con el entorno humano con el objetivo de conjuntamente solucionar cosas concretas. Ciertamente, la escuela debe partir del conocimiento lingüístico y de las capacidades desarrolladas en dichos contextos, pero la educación nunca podrá enseñar aquello que es informal, justamente porque es un contexto formal. Por eso, la escuela debe plantearse la enseñanza de la lengua en relación a los usos lingüísticos que en ella se realizan, usos que están directamente relacionados con sus actividades, las cuales son tareas de enseñanza-aprendizaje. La escuela debe promover el aprendizaje de los usos lingüísticos implicados en sus actividades (narración, argumentación, descripción, etc.) y, a la vez, asegurar que el lenguaje se domine como instrumento para conocer y aprender y, por tanto, debe trabajar explícitamente todos los aspectos relacionados con la comprensión del habla y de la escritura.

En general, lo formal y lo informal se ha relacionado con lo descontextualizado y lo contextualizado. Ciertamente, nuestro pensamiento no abona dicha distinción. En primer lugar, cuando nos referimos al uso del lenguaje la distinción entre lo descontextualizado y lo contextualizado es discutible. Por ejemplo, es evidente que en los contextos informales realizamos usos lingüísticos en los que predominan las referencias deícticas (contextualización extralingüística), mientras que en contextos formales predominan las referencias anafóricas (contextualización intralingǘstica), pero, en cualquier caso, se requiere del contexto (lo que está fuera del lenguaje o el mismo lenguaje) para poder comprender el sentido de los enunciados lingüísticos. De hecho, el progreso en el uso del lenguaje avanza en el sentido de la recontextualización más que en el de la descontextualización. En segundo lugar, la negociación del sentido de las producciones lingüísticas que realizan los interlocutores que están inmersos en la resolución de una tarea requiere, a la vez, de un contexto extralingǘstico y lingüístico para poderse realizar y, consecuentemente, tener acceso a las intenciones del otro. Por eso, no es posible enseñar lengua, bien sus usos informales, 
bien sus usos formales, al margen de un contexto que permita dicha negociación. La enseñanza de los usos formales de la lengua requiere obligatoriamente la contextualización de la tarea y de las producciones que la regulan.

\section{LA PSICOLINGUISTICA EVOLUTIVA Y LA ENSEÑANZA DE LA LENGUA}

Dicho lo anterior, una de las aportaciones que la psicología puede realizar a la enseñanza de la lengua remite al conocimiento del desarrollo de las capacidades implicadas en el uso del lenguaje. Es decir, si los humanos empleamos el lenguaje en situaciones significativas, bien para regular nuestra conducta en relación a la conducta de los otros, bien para regular nuestra conducta en relación a nosotros mismos, y los objetivos de la enseñanza de la lengua remiten, por tanto, al desarrollo de capacidades relativas al uso del lenguaje en situaciones colectivas o individuales, todo aquello que la psicolingüística evolutiva pueda aportar sobre el desarrollo de dichas capacidades será relevante para la planificación de los procesos de enseñanza-aprendizaje de la lengua.

En la actual psicolingǘstica evolutiva se reproducen los modelos epistemológicos sobre el desarrollo de las capacidades humanas. Ello aún es más cierto si entendemos el lenguaje (y no la comunicación) como una capacidad exclusivamente humana. De forma muy general, estos modelos remiten a la búsqueda de mecanismos psicológicos que explican el cambio evolutivo en el ámbito de lo individual o en el ámbito de la interacción social. Ciertamente, más allá de esta apreciación general, los modelos concretos presentan grandes diferencias entre ellos (incluso de orden epistemológico), pero para nuestra discusión esta distinción nos es útil.

De hecho, esta dicotomía remite en la mayoría de casos (al menos en el caso del lenguaje) a concepciones distintas sobre qué se desarrolla que se derivan de las distintas ideas que existen sobre cuál es, en último término, la dimensión lingüística (sintaxis, semántica, pragmática, etc.) sobre la que pivotan los demás aspectos que componen el lenguaje. Por ejemplo, es imposible entender los postulados generativistas al margen de la idea de creatividad de Chomsky y del rol que ocupa la sintaxis en dicha concepción. De la misma forma, no se puede entender alguna de las aproximaciones funcionalistas derivadas de la teoría de los actos de habla al margen de la idea del lenguaje como conducta lingüística. Por eso, para poder continuar con nuestra exposición es necesario aclarar qué queremos decir con capacidades lingüísticas relativas al uso del lenguaje.

Resulta fácil decir que estas capacidades remiten al conocimiento de los procedimientos implicados en el uso del lenguaje. Sin embargo, por baladí que pueda parecer, creo que la fórmula tiene miga. En primer lugar, implica una reflexión desde el propio uso del lenguaje. De hecho, la tradición en la lingüística y en la psicolingüística no es ésa. En general, los procedimientos implicados en el uso del lenguaje han sido descritos desde el lenguaje como objeto formal (sintaxis, morfología, léxico, etc.), independientemente de su puesta en práctica. Afortunadamente, en los últimos 25 años, la lingüística ha avanzado en este camino y hoy sabemos que el lenguaje en uso remite a complicados procesos que van más allá del dominio formal del código. Así, la distinción entre referencia proposicional y referencia discursiva es una buena distinción para, más allá de las 
raciones entre forma y significado, poder comprender las variaciones que se producen en el uso de las formas lingǘrsticas. Igualmente, el estudio de los proc sos (presuposiciones sobre el conocimiento y las actitudes del interlocutor, rec.nnocimiento de la adecuación de dichas presuposiciones, etc.) implicados en la noción de intersubjetividad aportan elementos para comprender la selección ๑. Ie los hablantes-oyentes hacen de su conocimiento formal del lenguaje. En cualquier caso, el intento de establecer los mecanismos formales y arbitrarios pertir: : ites en el uso del lenguaje ofrece una nueva perspectiva sobre las formas li. تísticas, radicalmente distinta en muchas ocasiones de las maneras más traci. - inales de abordar su descripción. En segundo lugar, esta perspectiva instruris al añade nuevas facetas para comprender el proceso de adquisición del lorguaje. Así, si nos situamos en el ámbito de la distinción entre la referencia 1): posicional y la referencia discursiva, podemos afirmar que la mayor parte de:la psicolingüística evolutiva ha asumido una concepción que reposa en el ca$\mathbf{r}^{\prime}$ 'Jer proposicional del lenguaje sin que los aspectos discursivos sean tomados ei. cuenta. En consecuencia, todo aquello relativo a la estratificación formal predomina sobre los planteamientos relativos a la estratificación funcional. Sin embargo, en los últimos años se ha abierto paso una concepción que evidencia las limitaciones de las investigaciones que remiten exclusivamente el estudio de la adquisición del lenguaje al ámbito proposicional. Por ejemplo, un aspecto que concierne a la referencia discursiva se refiere a las relaciones indexicales de tipo extrralingüístico o intralingüístico. Así, en el discurso de cualquier hablante no sólo se establecen relaciones de tipo signo-objeto (indexicalidad extralingüísticí: sino que también se establecen relaciones signo-signo (indexicalidad intrali. üística). En otras palabras, en el discurso, el habla se convierte también en un contexto de referencia. Las investigaciones evolutivas en psicolingüística muestran que los niños y las niñas dominan primero las relaciones indexicales extralingüísticas y, posteriormente, distinguen la función indexical intralingüística. Es decir, primero emplean aquello que está fuera del lenguaje (extralingüístico) como contexto de referencia y luego emplean el lenguaje como contexto de refere:cia (intralingǘŕstico). Ciertamente, tal y como señala Karmiloff-Smith, el domisio de la indexicalidad intralingüística presupone que el lugar de control de las oroducciones lingǘsticas deja de estar fuera del sujeto (relaciones signo-objeto) y.:'san a ser controladas por las categorías gramaticales que el sujeto construye el Il ámbito de lo individual.

En este planteamiento, la adquisición del lenguaje comporta un largo procesc in el que el sujeto no sólo incorpora nuevas formas lingüísticas, sino algo más importante: a lo largo del desarrollo aprende a usar de manera distinta las misnas formas lingüísticas. La adquisición del lenguaje no se contempla como un proceso lineal «in crescendo», sino como un proceso en $U$, enrevesado y comp.:-jo, en el que el sujeto progresivamente aprende a usar en el sentido de como lo usan los adultos aquello que ya usa. Por eso, aun situándonos en el ámbito din! código formal, aquello que nos permite comprender mejor los procedimiento implicados en el uso del lenguaje no es el análisis de la estructura lingüística, sino el análisis del discurso.

Sin embargo, como ya hemos señalado, la sustitución de la gramática estructural o generativa por una gramática textual o discursiva no mejoraría las cọsas en el ámbito de la enseñanza de la lengua. Es decir, el conocimiento consciente, por sí solo, de los mecanismos formales implicados en el uso del lenguaje 
ayuda poco a mejorar el dominio de las destrezas lingüísticas. De hecho, dicha práctica está en abierta contradicción con la perspectiva instrumental que estamos comentando.

Si consideramos que el uso del lenguaje se modifica en manos de los niños y las niñas a lo largo de su desarrollo, debemos preguntarnos sobre los mecanismos responsables de dicho cambio. Ciertamente, no existe una respuesta unívoca. Por el contrario, también en este ámbito se reproducen las discusiones sobre el predominio de lo individual o lo social. Sin embargo, no cabe duda de que el uso del lenguaje responde a un consenso social que, en último término, es cultural. Consenso que se manifiesta en el ámbito de la interacción social que es donde el lenguaje actúa y, en definitiva, donde se usa. Por eso, cualquier referencia al progreso en el uso del lenguaje (incluso aunque prime los aspectos individuales) no puede dejar de referirse a la interacción social, lugar en el que se manifiesta el uso del lenguaje. En consecuencia, es lícito pensar que el dominio de los procedimientos implicados en el uso del lenguaje se realiza cuando se utiliza el lenguaje en el ámbito de la interacción social. Desde esta perspectiva, se considera que no se puede aprender a usar algo al margen de sus contextos de uso. Es decir, de la misma forma que a conducir sólo se aprende conduciendo, los procedimientos lingüísticos sólo se pueden aprender utilizándolos. Ello comporta que, en la misma medida que al aprender a conducir una marca de coche concreta no sólo aprendemos a guiarlo sino también desarrollamos la capacidad para conducir que se transfiere a cualquier marca de coche, al aprender procedimientos linguísticos desarrollamos nuestra capacidad para usar el lenguaje. Capacidades que, ciertamente, se desarrollan desde el aprendizaje de lenguas concretas, pero que tienen mucho de común entre todos los hablantes de la especie humana, independientemente de la lengua o las lenguas que hablen y escriban.

Ello significa que aprendemos los usos lingǘsticos en la medida que hacemos cosas con el lenguaje. Pero, de la misma forma que la afirmación de que a conducir sólo se aprende conduciendo no significa que nos colocamos arriba de una cuesta, nos sentamos al volante y pedimos que nos den un empujón al coche, sino que aprendemos a conducir en la medida en que a nuestro lado se sienta alguien que ya sabe conducir y que, contingentemente, completa las conductas de conducir que no sabemos realizar solos, en la misma medida a hablar se aprende hablando y a escribir, escribiendo, siempre y cuando haya alguien que nos enseñe a hablar mejor de como hablamos y a escribir mejor de como escribimos. En este sentido, la enseñanza de la lengua debe partir de las producciones lingüísticas orales y escritas de los escolares y trabajar a partir de ellas, junto con modelos con los que los escolares puedan interactuar para progresivamente mejorar sus usos del lenguaje.

\section{DIDACTICA Y ENSEÑANZA DE LA LENGUA}

Hemos defendido que la psicolingüística evolutiva no puede prescribir los procesos de enseñanza-aprendizaje del lenguaje, aunque puede aportar conocimientos relativos al desarrollo de las capacidades relativas al uso del lenguaje. A la vez, creemos que los procedimientos implicados en el uso del lenguaje se adquieren desde sus contextos de uso y, por tanto, todo aquello que se refiere 
al estudio de los mecanismos de influencia educativa implicados en el aprendizaje de dichos procedimientos será también relevante para la planificación y mejora de los procesos de enseñanza-aprendizaje de la lengua.

La psicolingüística evolutiva señala que, en los contextos informales, los niños y las niñas reformulan sus usos lingüísticos en el ámbito de la interacción social y, por tanto, a lo largo de su desarrollo aprenden a usar de forma distinta aquello que ya usan. Sin embargo, en la educación existe (o debería existir) una clara intencionalidad de parte de los enseñantes referida a la mejora de los usos lingǘsticos. Es decir, el paralelismo de lo «natural» es poco relevante para el diseño y la planificación de la enseñanza de la lengua en el aula. Por eso, es necesario investigar en un doble sentido. En primer lugar, la psicología educativa debe avanzar en el estudio de los procesos de aprendizaje que son el resultado de procesos específicos de enseñanza (Coll, 1988). Ciertamente, esta investigación sólo se puede realizar en el marco del estudio de procesos de enseñanza-aprendizaje de contenidos concretos $\mathrm{y}$, por tanto, no remite al estudio de los procesos de aprendizaje de modo general sino a poseer un modelo teórico del proceso de enseñanza-aprendizaje en el que no sólo se contemple la actividad constructiva del alumno, sino también la actividad constructiva de la enseñanza. Es decir, si pensamos, siguiendo a Vigotski, que el alumno sólo construye conocimientos significativos en la medida en que aprende gracias al ajuste de la ayuda pedagógica del otro, las investigaciones sobre las estrategias didácticas de naturaleza constructivista son necesarias y relevantes para construir un modelo teórico de naturaleza constructivista de los procesos de enseñanza-aprendizaje. En segundo lugar, estrategia didáctica de naturaleza constructivista no significa metodología didáctica constructivista (Coll y al., 1992). Por el contrario, dicha estrategia se puede concretar en numerosas metodologías didácticas. Aquello que es importante investigar se refiere al tipo de ayuda pedagógica y a cómo se realiza su ajuste en relación a un contenido particular de aprendizaje que permite construir un determinado conocimiento a un alumno concreto. En el ámbito de la enseñanza de la lengua, esta investigación didáctica debería realizarse en relación a la enseñanza intencional de los procedimientos (en sentido amplio) implicados en el uso del lenguaje. Desgraciadamente, sabemos muy poco sobre este aspecto y son aún más las preguntas que las respuestas. Por ejemplo, deberíamos investigar sobre el rol de los modelos lingüísticos que se proporcionan de forma intencional a los alumnos y las alumnas con el objetivo de poseer un amplio banco de recursos didácticos de lengua oral y lengua escrita. Igualmente, se debería investigar sobre el rol de la reflexión metalingüística (cómo y cuándo hacerla) en la mejora del uso del lenguaje. En este sentido, uno de los problemas más importantes se refiere a la secuenciación de contenidos. Se ha repetido muchas veces que la mejora del uso del lenguaje responde a un proceso helicoidal y no lineal. Ello complica aún más las cosas en el ámbito de la secuenciación y, probablemente, la respuesta no se encuentre exclusivamente en los contenidos lingǘsticos, sino en las tareas que debe realizar el alumno en la escuela y el tipo de conocimientos que debe tener para resolverlas.

\section{CONCLUSIONES}

En este artículo hemos querido mostrar que la psicolingüística evolutiva no puede utilizarse de forma prescriptiva en el ámbito de la enseñanza de la len- 
gua. Ciertamente, la historia de las relaciones entre enseñanza de la lengua y psicología no es esa. Por el contrario, durante años y, aún en la actualidad, tanto en la enseñanza de segundas lenguas como de la propia lengua, la psicología $y$, especialmente, la psicolingüística ha sido el eje rector de muchas de las propuestas didácticas. La existencia de un cierto paralelismo entre el proceso «natural» de adquisición del lenguaje y el que se da en la escuela a través del proceso de aprendizaje del lenguaje ha tenido mucho peso en el pensamiento y las creencias de un sector del profesorado y se ha traducido en prácticas, muchas veces espontaneístas, arropadas en la función comunicativa del lenguaje, que en vez de promover aprendizajes intencionales relativos a la mejora en el uso del lenguaje se limitaban a proponer actividades lingüísticas cuya realización se convertía en un fin en sí mismo. Por eso, hemos propuesto que las preguntas y las respuestas relevantes en el ámbito de la enseñanza de la lengua no pueden provenir de la psicolingüística sino del estudio de los mecanismos de influencia educativa que promueven el aprendizaje de los procedimientos implicados en el uso del lenguaje. Evidentemente, esta tarea no entra en el campo de la psicolingǘstica, sino de la psicología educativa y de la didáctica de la lengua, lo cual no significa que determinados conocimientos psicolingüísticos no sean pertinentes para comprender mejor los procesos de comprensión y producción de la lengua oral y de la lengua escrita. Conocimientos que no se deben desdeñar, sino integrar en los postulados teóricos y metodológicos que guíen dicha investigación.

La psicología educativa y la didáctica de la lengua convergen, por tanto, en el objeto de estudio. De una parte, la psicología educativa debe avanzar en la construcción de un modelo sobre el proceso de enseñanza-aprendizaje que contemple simultáneamente el proceso constructivo del alumno y el proceso constructivo de la enseñanza. Es decir, un modelo que sea capaz de dar cuenta del tipo y el ajuste de la ayuda pedagógica que permite que un alumno determinado construya un tipo particular de conocimiento o aprenda a usar una habilidad determinada. De la otra, dicho modelo sólo se puede construir en la medida en que se estudien y se investiguen procesos de enseñanza-aprendizaje de contenidos concretos (conceptos, procedimientos, valores, hechos, etc.) y, por tanto, en el ámbito de la enseñanza de la lengua, se debería proponer lo mismo que ya proponen bastantes didactas de la lengua.

La elaboración de criterios que permitan a los profesores y las profesoras diseñar y planificar procesos efectivos de enseñanza-aprendizaje de la lengua es una tarea común que deben emprender conjuntamente psicólogos educativos y didactas de la lengua, para los cuales el conocimiento psicolingüístico, como otros conocimientos (sociolingüística, psicología de la instrucción, etc.), creo que es pertinente siempre y cuando se delimite claramente su ámbito de aplicación.

\section{Referencias}

CAMPs, A. (1990). Los objetivos lingüísticos de la educación. En M. Siguan (ed.). Lengua del alumno, lengua de la escuela. Barcelona: PPU.

Cols, C. (1988). Conocimiento psicológico y práctica educativa. Barcelona: Barcanova.

Coll, C.; Colomina, R.; Onrubia, J., y Rochera, M. J. (1992). Actividad conjunta y habla: Una aproximación al estudio de los mecanismos de influencia educativa. Infancia y Aprendizaje, 59.60, 189-232.

Сномsкy, N. (1988). El lenguaje y los problemas del conocimiento. Madrid: Visor. 


\section{Extended Summary}

In this paper we have wanted to show that developmental psycholinguistics cannot be used to prescribe within the field of language teaching. Indeed, that is not the history of relations between language teaching and psychology. On the contrary, for years and even today, both in first and second language teaching, psychology and specially psycholinguistics has been the axis of many didactic proposals. The existence of a certain parallelism between the 'natural' process of language aquisition and the process which takes place at school through the language learning process has had a lot of influence on the thoughts and beliefs of some teachers. This in turn has been translated into frequently spontaneous teaching styles cloaked in the communicative function of language, which instead of promoting intentional learning, related to improving the use of language, are limited to the proposal of linguistic activities whose undertaking became an end in itself. For this reason we have proposed that those questions and answers which are relevant within the field of language teaching cannot come from psycholinguistics but from the study of the mechanisms of educational influence promoted by learning the procedures involved in the use of language. Evidently, this task does not belong to the field of psycholinguistics, but to the fields of Educational Psychology and Language Didactics. This does not mean that a certain psycholinguistic knowledge is not relevant for a better understanding of comprehension and production processes oral and written language. This knowledge must not be rejected, but intergrated in the theoretical and methodological postulates that guide the research.

Educational Psychology and Language Didactics therefore converge in their object of study. On the one hand Educational Psychology must advance in constructing a model about the teaching-learning process that simultaneous envisages the student's constructive process and the teaching process. In other words, a model that is capable of accounting for the type and adjustment of help from the teacher which allows a specific student to construct a particular type of knowledge or to learn to use a specific skill. On the other hand, such model may only be constructed insofar as teaching-learning processes for specific contents (concepts, procedures, values, facts, etc.) are studied and investigated. Therefore, in the field of language teaching the same should be proposed as already quite a few language didactics are proposing.

Elaborating criteria allowing teachers to design and plan efective language teaching-learning processes is a common task which both Educational Psychologists and language didactics professionals should undertake. To this end psycholinguistic knowledge, together with knowledge from other areas (Sociolinguistics, Instructional Psychology, etc.), are relevant whenever its area of practice is clearly specified. 